\title{
Tratamento oncológico: desafios e perspectivas na comunicação da enfermagem: revisão integrativa
}

\author{
Cancer treatment: challenges and perspectives in nursing communication: integrative review \\ Tratamiento del cáncer: desafíos y perspectivas en la comunicación de enfermería: \\ revisión integrativa
}

Leidiany Gomes Moreira ${ }^{1 *}$, Janaina Gonçalves Schmidt de Paula ${ }^{2}$, Renata Angélica Ferreira de Oliveira², Wilkner Gustavo de Oliveira Aguiar², José Mansano Bauman², André Luiz Gomes Carneiro², Priscila Bernardina Miranda Soares ${ }^{3}$, Carla Silvana Oliveira e Silva², Claudiana Donato Bauman².

\section{RESUMO}

Objetivo: Apontar os principais desafios e perspectivas da enfermagem oncológica na comunicação de notícias para o paciente oncológico e seus familiares. Métodos: Trata-se de uma revisão integrativa que atendeu as recomendações do PRISMA. As bases de dados utilizadas foram: Bireme, Medline, Lilacs, BDENF e SciELO, via descritores: "oncology nursing", "nursing", "oncology" e "health communication", no período de 2011 a 2021. Identificou-se 274 estudos, e após os critérios de inclusão, 9 investigações compuseram a amostra. Resultados: $77,7 \%$ das investigações apontaram que a falta de treinamento e capacitações transculturais, a ausência de sistematização, a falta de habilidades adquiridas na graduação, a ineficácia de esforços colaborativos, a aproximação nos padrões éticos, humanos e assistenciais se relacionaram aos principais desafios. Quanto às perspectivas, ressaltou-se o relevante papel humanitário no tratamento oncológico, assim como a capacidade e aptidão da enfermagem para a comunicação prognóstica com o paciente e seus familiares. Considerações finais: A equipe de enfermagem oncológica é essencial na comunicação de qualidade, entretanto, maior autonomia aos profissionais, investimentos em treinamentos e sistematizações ainda são um grande desafio. A atuação da equipe se apresentou como fundamental na qualidade da informação e humanização, abalizando perspectivas positivas e abrangências profissionais promissoras para o desenvolvimento de habilidades específicas.

Palavras-chave: Enfermagem oncológica, Oncologia, Comunicação em saúde.

\begin{abstract}
Objective: Point out the main challenges and perspectives of cancer nursing in communicating news to cancer patients and their families. Methods: This is an integrative review that met the PRISMA recommendations. The databases used were: Bireme, Medline, Lilacs, BDENF and SciELO, via descriptors: "oncology nursing", "nursing", "oncology" and "health communication", from 2011 to 2021. 274 studies were identified, and after the inclusion criteria, 9 investigations composed the sample. Results: $77,7 \%$ of the investigations indicated that the lack of training and cross-cultural qualifications, the absence of systematization, the lack of skills acquired in graduation, the ineffectiveness of collaborative efforts, the approximation of ethical, human and care standards were related to the main challenges. As for the perspectives, the relevant humanitarian role in cancer treatment was highlighted, as well as the capacity and aptitude of nursing for prognostic communication with patients and their families. Final considerations: The oncology nursing team is essential in quality communication, however, greater autonomy for professionals, investments in training and systematization are still a major challenge. The team's performance was shown to be fundamental in the quality of information and humanization, supporting positive perspectives and promising professional ranges for the development of specific skills.
\end{abstract}

Keywords: Oncology nursing, Oncology, Health communication.

${ }^{1}$ Faculdade Batista Grupo Ipemig, Belo Horizonte - MG. *leidianyg@hotmail.com

2 Universidade Estadual de Montes Claros (UNIMONTES), Montes Claros - MG.

3 Hospital Oncovida Dia, Montes Claros - MG. 


\section{RESUMEN}

Objetivo: Señalar los principales desafíos y perspectivas de la enfermería oncológica en la comunicación de noticias a los pacientes con cáncer y sus familias. Métodos: Se trata de una revisión integrativa que cumplió con las recomendaciones de PRISMA. Las bases de datos utilizadas fueron: Bireme, Medline, Lilacs, BDENF y SCIELO, mediante descriptores: "enfermería oncológica", "enfermería", "oncología" y "comunicación en salud", de 2011 a 2021. Se identificaron 274 estudios. criterios de inclusión, 9 investigaciones componen la muestra. Resultados: el $77,7 \%$ de las investigaciones indicaron que la falta de formación y calificaciones transculturales, la ausencia de sistematización, la falta de competencias adquiridas en la graduación, la ineficacia de los esfuerzos colaborativos, la aproximación de estándares éticos, humanos y de atención estaban relacionados con los principales retos. En cuanto a las perspectivas, se destacó el papel humanitario relevante en el tratamiento del cáncer, así como la capacidad y aptitud de la enfermería para la comunicación pronóstica con los pacientes y sus familiares. Consideraciones finales: El equipo de enfermería oncológica es fundamental en la calidad de la comunicación, sin embargo, la mayor autonomía de los profesionales, las inversiones en formación y sistematización siguen siendo un gran desafío. El desempeño del equipo se mostró fundamental en la calidad de la información y la humanización, apoyando perspectivas positivas y rangos profesionales prometedores para el desarrollo de competencias específicas.

Palabras clave: Enfermería oncológica, Oncología médica, Comunicación en salud.

\section{INTRODUÇÃO}

O Instituto Nacional do Câncer (INCA) evidenciou que a incidência do câncer tem aumentado a cada ano, sendo responsável por uma das principais causas de mortalidade no mundo, assim como a morbidade hospitalar que se conota em um cenário preocupante para gestores em saúde e vigilância epidemiológica, uma vez, que a evolução desacelerada dificulta a análise da ocorrência, a distribuição e a evolução das doenças, fatores essenciais para as tomadas de decisão em saúde pública (INCA, 2021).

O Instituto ressalta que de acordo com as estatísticas, haverá um aumento de 625 mil casos de brasileiros com câncer a cada ano do triênio 2020-2022. Mesmo com recursos e inovações disponíveis que podem curar ou prolongar a sobrevivência dos indivíduos, ainda existem muitas dificuldades relacionadas à patologia, por se tratar de uma doença complexa com prognósticos que se relacionam individualmente para cada ser (SILVA MF e BEZERRA MLR, 2020).

A duração da doença depende de diversos fatores e afeta a vida do indivíduo em diferentes dimensões (biopsicossocial e espiritual) e algumas vezes, de formas irreparáveis. Entretanto, o resultado do tratamento pode ser fortalecido mediante o acompanhamento qualificado por parte de uma equipe multiprofissional (FALCÃO VM, et al., 2020).

Após a transmissão da notícia de um câncer a um paciente, a família também adoece, assim como as pessoas mais próximas, pois o mesmo traz mudanças, inseguranças e incertezas de um futuro para todos envolvidos naquele ciclo. Contudo é o apoio da família e sociedade (aliados aos tratamentos complementares) que vão ditar a evolução do paciente, pois permitem a que a segurança e a esperança de que tudo vai passar (SALCI MA e MARCON SS, 2011).

Na rotina dos profissionais da saúde a comunicação interpessoal é algo que se faz presente. A notícia difícil caracteriza-se como algo natural na oncologia, e define-se como qualquer informação de conteúdo negativo que resulte na vida do paciente e ou familiar. $O$ apoio multidisciplinar contínuo, assim como a proximidade nas relações interpessoais, pode favorecer ações de saúde e educação eficientes durante o tratamento oncológico. Neste contexto, o profissional da enfermagem é o membro da equipe que mais tempo mantém contato o paciente, sendo muitas vezes o primeiro a identificar os efeitos indesejáveis (OLIVEIRA AEG e CURY VE, 2016).

Conhecer profundamente os aspectos, os sinais e sintomas, tipos de tratamentos, efeitos colaterais e os cuidados de enfermagem que podem ser prestados, são necessidades dos enfermeiros oncológicos, uma vez que como membros da equipe de saúde, assumem função vital na recuperação do paciente, assim como podem atuar nesse canal de comunicação (SOUZA FLN, et al., 2021). 
Sob essa ótica, a humanização da saúde e a compreensão do respeito à unicidade de cada pessoa, permite a personalização da assistência e a transformação das práticas de saúde pautadas no cuidado relacionando o sofrimento seguido da doença, a ponto do reconhecimento da dor e aflição humana (WALDOW VR e BORGES RF, 2011).

A enfermagem oncológica é capacitada para prestar uma assistência individualizada ao paciente com câncer, contribuindo para a humanização no processo da notícia a ser repassada e colaborando com a qualidade de vida do paciente. $O$ acolhimento à família também se torna imprescindível, tanto no diagnóstico, como no tratamento, reabilitação ou nos cuidados paliativos. O entendimento do processo, a amenização das dores e sofrimentos (físicos e emocionais) provocados pela doença, alinhados à uma equipe de enfermagem capacitada pode favorecer um ambiente menos carregado (mais leve) para todos envolvidos neste ciclo (SILVA RCV e CRUZ EA, 2011).

O presente estudo objetivou revisar e apontar os principais desafios e perspectivas da enfermagem oncológica na comunicação de notícias para o paciente com câncer e seus familiares.

\section{MÉTODOS}

Trata-se de uma revisão integrativa que atendeu as recomendações do Relatório Preferenciais para Revisões Sistemáticas e Meta-análises (PRISMA), pautada em estudos e intervenções científicas com classificação de evidências, fundamentadas na proposta de Melnyk BM, Fineout-Overholt E (2005).

Foram qualificadas as evidencias em sete níveis. No nível 1 são as originadas de revisão sistemática ou metanálise de relevantes ensaios clínicos randomizados; no nível 2, evidências decorrentes de pelo menos um ensaio clínico controlado bem elaborado; no nível 3, evidências adquiridas de ensaios clínicos bem orientado sem randomização; no nível 4, evidências advindas de estudos de coorte e de caso-controle; nível 5 , evidências que vieram de revisões sistemáticas de estudos descritivos e qualitativos; nível 6 , evidências vindas de um único estudo descritivo e qualitativo; no nível 7, evidências provindas de opinião de autoridades e/ou relatório de comitês de especialistas (GALVÃO CM, 2007).

A pergunta norteadora do presente estudo, se concentrou na compreensão dos principais desafios da enfermagem oncológica, assim como as perspectivas na comunicação de notícias para o paciente e seus familiares. As bases de dados utilizadas para a busca ocorreram através da biblioteca virtual em Saúde (BVS), incluindo-se o Centro Latino-Americano e do Caribe de Informação em Ciências da Saúde (BIREME), o Sistema Online de Busca e Análise de Literatura Médica (MEDLINE), a Literatura Latino-Americana e do Caribe em Ciências da Saúde (LILACS), a Base de Dados de Enfermagem (BDENF) e a Scientific Electronic Library Online (SciELO), no período de abril de 2021. Os operadores booleanos foram o "AND" e o "OR". Os seguintes descritores foram utilizados, de acordo com Descritores em Ciências da Saúde (DECs) e Medical Subject Headings (MeSH): Oncology Nursing, nursing, oncology, e Health Communication, publicados no período de 2011 a 2021. Foram utilizados artigos nesse recorte temporal a fim de compilar um maior número de informações sobre o tema para que, assim, o artigo fosse mais fidedigno ao objetivo proposto.

Como critério de inclusão optou-se por estudos disponíveis na íntegra, em português, inglês e espanhol. Foram identificados 274 estudos com a descrição acima. Após a análise, a amostra final foi composta por nove artigos científicos, que responderam em suas conclusões, de forma específica a pergunta norteadora.

\section{RESULTADOS}

De acordo com a Figura 1 é possível visualizar o processo de seleção dos artigos a partir da busca nas bases de dados citadas acima. Como exemplificado a seguir, a partir de uma busca inicial foram encontrados 1329 artigos, e, após a utilização dos filtros relacionados a data e idiomas o número de artigos se reduziu para 274. Após isso, foi realizada uma leitura dos resumos dos 274 estudos e apenas 23 deles se enquadravam nos objetivos pesquisados. No entanto, 14 desses artigos foram excluídos devido a duplicidade, dissonância com o tema estudado bem como tangenciamento, restando assim 9 artigos que foram incluídos nessa revisão integrativa. 
Figura 1 - Escolha dos artigos.

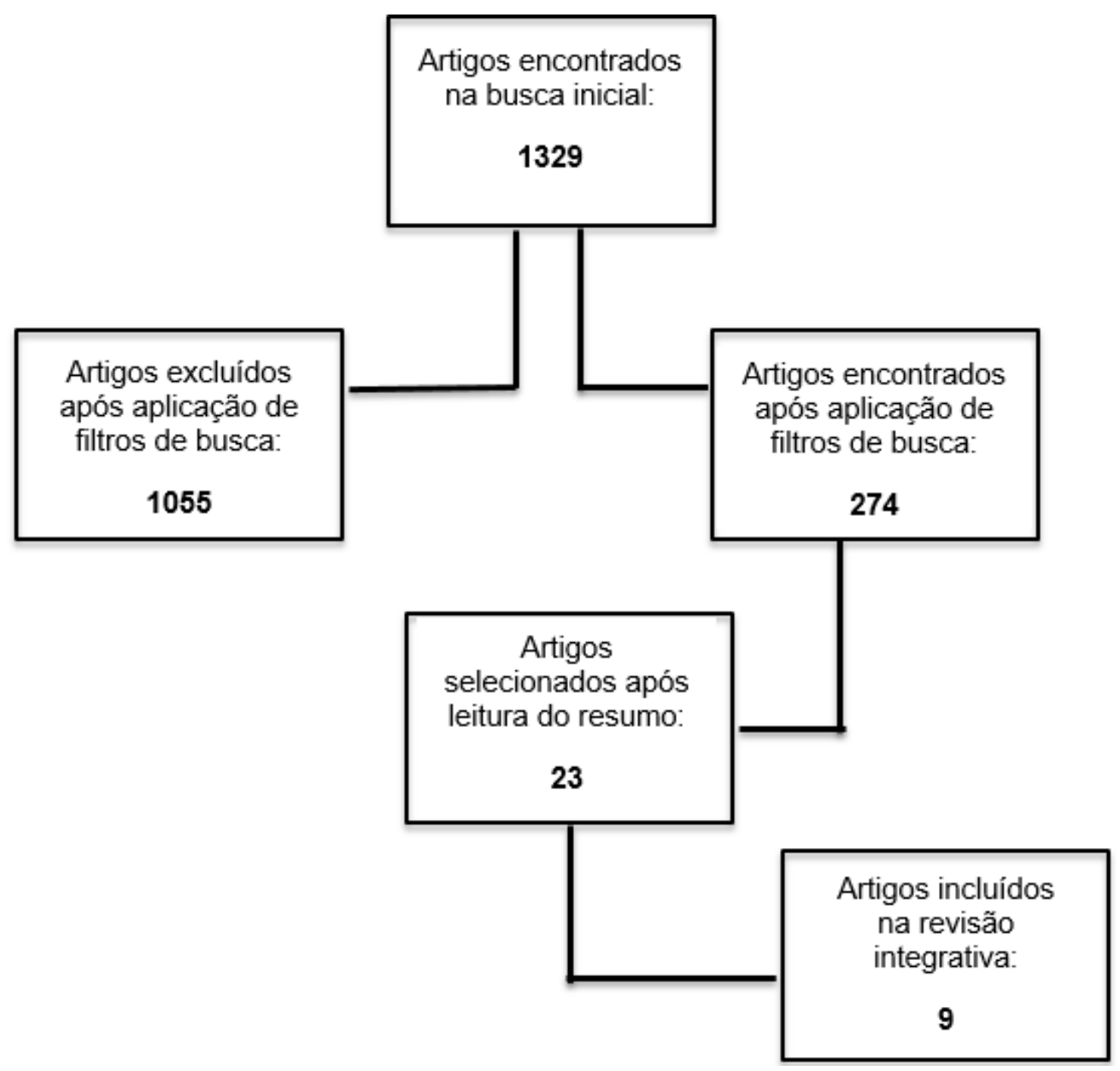

Fonte: Moreira LG, et al., 2021.

Os 9 estudos incluídos, serão apresentados de acordo com o número correspondente, ano de publicação, autoria, título, periódico, nível de evidência, objetivo e conclusão (Quadro 1). 
Quadro 1 - Artigos selecionados para a revisão integrativa.

\begin{tabular}{|c|c|c|c|c|c|c|}
\hline № & AUTORIA & TÍTULO & PERÍODICO & NE & OBJETIVO & CONCLUSÃO \\
\hline 1 & $\begin{array}{l}\text { MENDES JLV, } \\
\text { et al., } 2020\end{array}$ & $\begin{array}{l}\text { Importância da } \\
\text { comunicação para uma } \\
\text { assistência de qualidade: } \\
\text { Uma revisão Integrativa. }\end{array}$ & $\begin{array}{l}\text { Brazilian Journal of } \\
\text { Surgery \& Clinical } \\
\text { Research. }\end{array}$ & 5 & $\begin{array}{l}\text { Caracterizar a produção científica dos últimos } \\
\text { cinco anos acerca da importância da } \\
\text { comunicação para a assistência prestada pela } \\
\text { enfermagem. }\end{array}$ & $\begin{array}{l}\text { O processo da comunicação embora seja crucial para a área da } \\
\text { enfermagem na atuação dos profissionais e envolvidos, } \\
\text { apresenta muitas deficiências que corroboram para riscos tanto } \\
\text { para o profissional quanto para a equipe e clientes, sejam eles } \\
\text { o próprio paciente ou a família, gerando insatisfações, erros, } \\
\text { atritos entre a equipe e o profissional e até mesmo com o } \\
\text { cliente, prejudicando na realização da terapêutica. }\end{array}$ \\
\hline 2 & $\begin{array}{l}\text { WITTENBERG } \\
\text { E, et al., } 2019\end{array}$ & $\begin{array}{l}\text { Communication Training: } \\
\text { Needs Among Oncology } \\
\text { Nurses Across the Cancer } \\
\text { Continuum. }\end{array}$ & Clin J Oncol Nurs & 5 & $\begin{array}{l}\text { Este estudo relata as necessidades de } \\
\text { treinamento em comunicação do enfermeiro } \\
\text { oncológico, visto que desconfortos e } \\
\text { dificuldades comunicativas trazem diversos } \\
\text { prejuízos. }\end{array}$ & $\begin{array}{l}\text { A formação dos enfermeiros é necessária e o desenvolvimento } \\
\text { do currículo deve ser congruente com os papéis comunicativos } \\
\text { institucionais dos enfermeiros oncológicos. }\end{array}$ \\
\hline 3 & $\begin{array}{l}\text { ARAÚJO BN, et } \\
\text { al., } 2017\end{array}$ & $\begin{array}{l}\text { Acolhimento do enfermeiro } \\
\text { aos familiares de } \\
\text { portadores de câncer: a } \\
\text { percepção do familiar. }\end{array}$ & $\begin{array}{l}\text { Revista Saúde e } \\
\text { Desenvolvimento }\end{array}$ & 5 & $\begin{array}{l}\text { Compreender o acolhimento que é prestado } \\
\text { pelo enfermeiro aos familiares de portadores } \\
\text { de câncer na percepção do familiar. }\end{array}$ & $\begin{array}{l}\text { Foi possível compreender como o acolhimento transforma o } \\
\text { modo de atender pacientes e familiares durante o tratamento } \\
\text { oncológico, pois observou-se a importância que possui na vida } \\
\text { dos pacientes/familiares, bem como a diferença que traz. }\end{array}$ \\
\hline 4 & $\begin{array}{l}\text { FONTES CMB, } \\
\text { et al., } 2017\end{array}$ & $\begin{array}{l}\text { Communicating bad news: } \\
\text { an integrative review of the } \\
\text { nursing literature. }\end{array}$ & $\begin{array}{c}\text { Revista Brasileira } \\
\text { Enfermagem }\end{array}$ & 5 & $\begin{array}{l}\text { Descrever como se estabelece o processo de } \\
\text { comunicação de más notícias e identificar } \\
\text { como o enfermeiro pratica a comunicação de } \\
\text { más notícias. }\end{array}$ & $\begin{array}{l}\text { Percebeu-se na realidade de dar más notícias a forte influência } \\
\text { cultural no trabalho da enfermagem em países desenvolvidos, } \\
\text { onde possuem maior autonomia para discutir as notícias com } \\
\text { os pacientes. Porém, é notável que a questão cultural influencia } \\
\text { o enfermeiro, tornando-o um profissional submisso e não } \\
\text { autônomo no comunicar notícias aos pacientes e suas famílias. }\end{array}$ \\
\hline 5 & $\begin{array}{c}\text { ZUCA A, et al., } \\
2017\end{array}$ & $\begin{array}{l}\text { The first step in ensuring } \\
\text { patient-centred quality of } \\
\text { care: ask the patient. }\end{array}$ & $\begin{array}{c}\text { Eur J Cancer Care } \\
\text { (Engl). }\end{array}$ & 5 & $\begin{array}{l}\text { Determinar se os pacientes foram } \\
\text { questionados pela equipe da clínica no centro } \\
\text { de tratamento sobre seus sintomas físicos e } \\
\text { emocionais, necessidades de informações } \\
\text { para permitir a tomada de decisões, } \\
\text { preferências de envolvimento na tomada de } \\
\text { decisões, envolvimento de familiares e amigos } \\
\text { e agendamento de consultas. }\end{array}$ & $\begin{array}{l}\text { Parece que a maioria dos pacientes ambulatoriais de oncologia } \\
\text { médica foi questionada sobre as preferências e necessidades } \\
\text { na maioria dos aspectos centrados no paciente. No entanto, há } \\
\text { espaço para melhorias em solicitar mais da metade dos } \\
\text { pacientes de oncologia médica no ambiente avaliado. No } \\
\text { entanto, perguntar é apenas o primeiro passo. Em seguida, } \\
\text { precisamos garantir que os pacientes recebam ajuda } \\
\text { subsequentemente e que a ajuda seja consistente com suas } \\
\text { necessidades e alivia o sofrimento. }\end{array}$ \\
\hline
\end{tabular}




\begin{tabular}{|c|c|c|c|c|c|c|}
\hline № & AUTORIA & TÍTULO & PERÍODICO & NE & OBJETIVO & CONCLUSÃO \\
\hline 6 & $\begin{array}{l}\text { WEBER O, et } \\
\text { al., } 2016\end{array}$ & $\begin{array}{l}\text { Cross-Cultural } \\
\text { Communication in } \\
\text { Oncology: Challenges and } \\
\text { Training Interests. }\end{array}$ & Oncol Nurs Forum. & 5 & $\begin{array}{l}\text { Realizar pesquisas com enfermeiras } \\
\text { oncologistas e oncologistas sobre as } \\
\text { dificuldades em cuidar de pacientes de } \\
\text { diferentes línguas e culturas. }\end{array}$ & $\begin{array}{l}\text { A necessidade de treinamento transcultural é alta entre os } \\
\text { médicos oncológicos, especialmente entre os enfermeiros. }\end{array}$ \\
\hline 7 & $\begin{array}{l}\text { QIU WL, et al., } \\
2013\end{array}$ & $\begin{array}{l}\text { Requests for health } \\
\text { education from Chinese } \\
\text { cancer patients during their } \\
\text { recovery period: a cross- } \\
\text { sectional study. }\end{array}$ & J Cancer Educ. & 5 & $\begin{array}{l}\text { Determinar a prevalência de solicitações de } \\
\text { pacientes pós-tratamento por informações de } \\
\text { saúde sobre câncer na China. A pesquisa } \\
\text { abordou o que tipos de informações que os } \\
\text { pacientes realmente queriam, mas não } \\
\text { receberam de seus cuidadores. }\end{array}$ & $\begin{array}{l}\text { O estudo mostra que um modelo de intervenção de educação } \\
\text { em saúde durante o período de recuperação é necessário, mas } \\
\text { requer esforços colaborativos dos profissionais de saúde do } \\
\text { hospital, familiares e recursos da comunidade para realizar o } \\
\text { cuidado holístico. }\end{array}$ \\
\hline 8 & $\begin{array}{l}\text { MOORE PM, et } \\
\text { al., } 2013\end{array}$ & $\begin{array}{l}\text { Communication skills } \\
\text { training for healthcare } \\
\text { professionals working with } \\
\text { people who have cancer. }\end{array}$ & $\begin{array}{c}\text { Cochrane Database } \\
\text { Syst Ver. }\end{array}$ & 1 & $\begin{array}{l}\text { O objetivo deste artigo é avaliar se o } \\
\text { treinamento de habilidades de comunicação } \\
\text { (CST) é eficaz mudar o comportamento dos } \\
\text { profissionais de saúde (HCPs) no trabalho no } \\
\text { tratamento do câncer e melhorar o bem-estar, } \\
\text { a saúde do paciente, o estado e a satisfação } \\
\text { do profissional de saúde. }\end{array}$ & $\begin{array}{l}\text { Treinamento de habilidades de comunicação para profissionais } \\
\text { de saúde (HCPs) que trabalham no tratamento do câncer } \\
\text { usando métodos de educação experiencial centrados no aluno } \\
\text { por facilitadores experientes, pode resultar em melhorias em } \\
\text { algumas habilidades de comunicação, particularmente empatia, } \\
\text { e pode ajudar HCP a ser menos propenso a fornecer fatos } \\
\text { apenas sem individualizando suas respostas às emoções do } \\
\text { paciente ou oferecendo apoio. Não está claro se as } \\
\text { competências adquiridas pelos profissionais de saúde são } \\
\text { mantidas a longo prazo. Além disso, não está claro qual tipo, } \\
\text { duração e intensidade do CST é mais eficaz e se os workshops } \\
\text { de consolidação podem melhorar o impacto do CST. O CST } \\
\text { parece ter poucos benefícios mensuráveis para a saúde mental } \\
\text { ou física e satisfação das pessoas com câncer e não parece } \\
\text { reduzir o "esgotamento" nos profissionais de saúde. }\end{array}$ \\
\hline 9 & $\begin{array}{l}\text { MEYSTRE C., } \\
2013\end{array}$ & $\begin{array}{l}\text { Working alliance in } \\
\text { communication skills } \\
\text { training for oncology } \\
\text { clinicians: a controlled trial. }\end{array}$ & $\begin{array}{l}\text { Patient Educ } \\
\text { Couns. }\end{array}$ & 2 & $\begin{array}{l}\text { O objetivo deste estudo foi avaliar o impacto } \\
\text { do treinamento de habilidades de } \\
\text { comunicação (CST) na aliança de trabalho e } \\
\text { identificar elementos comunicacionais } \\
\text { específicos relacionados à aliança de trabalho. }\end{array}$ & $\begin{array}{l}\text { Os resultados destacam o papel da comunicação específica } \\
\text { comportamentos e seu impacto na construção de alianças. } \\
\text { Novos estudos são necessários para confirmar esses achados, } \\
\text { especialmente com pacientes reais, que os médicos já } \\
\text { conhecem, para refletir a realidade clínica. }\end{array}$ \\
\hline
\end{tabular}

Fonte: Moreira LG, et al., 2021. 


\section{DISCUSSÃO}

Relacionando a comunicação de notícias acerca do tratamento oncológico com o paciente, família e equipe clínica, entendeu-se que o diálogo é primordial no sentido da prevenção de danos graves, assim como resultados negativos e sofrimento antecipado, que poderão ser constituídos na trajetória oncológica. Várias barreiras manifestadas através das conclusões foram relatadas nos artigos incluídos nessa investigação. Os principais desafios se concatenaram em empecilhos inibidores significativos, como discrepâncias de poder, além da falta de convicção da equipe de enfermagem, medo e despreparo profissional para a realização de tarefas relacionadas à comunicação prognóstica (MENDES JLV, et al., 2020).

A comunicação considerada eficiente é caracterizada como um processo que oferece robustez à simples palavras. Compreende a preocupação compartilhada e o reconhecimento daquilo que está sendo informado para o próximo. Sisk BA, et al. (2020), evidenciaram que a maioria das dificuldades na comunicação se associam à incipiência da motivação por parte da equipe de enfermagem, geradas pelas insatisfações e insuficiência de recursos humanos e estruturais (BORDIGNON M, et al., 2015).

No estudo classificado como número 1 nos quadros acima, foi apresentada a produção científica dos últimos cinco anos, e evidenciada a importância da comunicação para a assistência prestada pela enfermagem. Os autores ressaltaram que o processo da comunicação, embora crucial para a área da enfermagem, apresentou diversas deficiências e afirmaram que são múltiplos os riscos tanto para os profissionais quanto para a equipe e clientes. Insatisfações, erros e atritos entre a equipe e o profissional, assim como o profissional e o cliente, são extremamente prejudiciais na realização da terapêutica e propedêutica (MENDES JLV, et al., 2020).

De acordo com a constatação, o estudo evidenciou que a comunicação é um elemento essencial e falhas nesse trajeto trazem prejuízos significativos tanto para a equipe quanto para o paciente. A comunicação ineficaz é um dos principais fatores para a ocorrência de erros ou danos adversos (EA), que tem uma taxa de mortalidade quarenta vezes maiores do que em um acidente de trânsito (PENA MM e MELLEIRO MM, 2018).

Evidenciam-se também nos estudos barreiras e critérios facilitadores da comunicação de notícias negativas na atenção básica de saúde. Nesse sentido, foi enfatizado que um dos problemas enfrentados pela equipe de enfermagem encontra-se na alta demanda de usuários, o alto fluxo de pessoas e a densidade de clientes para os atendimentos (SISK BA, et al., 2020). Essa questão impõe que enfermeiros, cumpram as atividades relacionadas às demandas, integrando um fator dificultador da atenção e dedicação à comunicação.

Outra questão levantada, se encontra na dimensão psicológica de quem leva as notícias ruins, pois em função da carga negativa, muitas vezes não se encontram aptos para lidarem com a maneira que essas notícias repercutem neles próprios, o que pode levar ao comprometimento da comunicação eficaz (ARAÚJO BN, et al., 2017).

Outro ponto que se apresentou como barreira, foi a lida com portadores de deficiências, principalmente auditivas e visuais. Profissionais na área da saúde apresentam um déficit na interpretação e domínio de formas diferentes de comunicação, como a LIBRAS. A dificuldade em entender e se expressar por meio dessa linguagem pode gerar ambiguidades e desentendimento, entre o Enfermeiro e família/paciente. A equipe de enfermagem ainda está, na sua maioria, despreparada para atender a população com necessidades especiais, o que interfere diretamente na qualidade do cuidado prestado e contribui para a resistência apresentada por esses indivíduos quanto à procura dos serviços de saúde e adesão ao tratamento correto (WITTENBERG E, et al., 2019).

Quanto as questões facilitadoras, os autores ressaltaram a importância da informática na enfermagem, que é descrita como uma especialidade desde 1985, englobando processos tecnológicos de informatização, representa um aspecto importante para a enfermagem nos dias atuais. Os computadores são utilizados pelos enfermeiros para diversas funções, como: comunicar e recuperar dados para a tomada de decisões. Gerar informações para o controle e qualidade de assistência. Orientar pacientes sobre cuidados em saúde geral e específicos (MIRANDA GM et al., 2019). Foi destacado o MobiNurse, um protótipo de sistema de informação utilizado no atendimento à domicílio. 
A comunicação eficaz e o trabalho em equipe são aspectos favoráveis para a realização das notificações. Dados da pesquisa corroboram com a literatura no tocante ao trabalho em equipe e a comunicação serem elementos chave para uma cultura segura (PENA MM e MELLEIRO MM, 2018).

De acordo com os resultados apresentados, dois estudos apontaram que a equipe de enfermagem é essencial na comunicação prognóstica e possui um relevante papel na qualidade da informação e humanização do processo (ARAÚJO BN, et al., 2017; SISK BA, et al., 2020). Entretanto um dos estudos demonstrou que a equipe de enfermagem é frequentemente excluída da comunicação prognóstica, tornando necessária uma articulação multidisciplinar onde a equipe de enfermagem é incorporada diretamente na comunicação prognóstica familiar, tornando-se uma comunicação eficaz (MENDES JLV, et al., 2020).

Os estudos concluíram, que tanto a equipe de enfermagem quanto os demais membros da equipe clínica, necessitam de capacitação, principalmente na humanização e assistência, para que possam acompanhar e fornecer conforto emocional, além do alivio da dor. Partindo de um elo com o paciente/família, é enaltecido no estudo, uma forma positiva da inserção dos procedimentos terapêuticos no cotidiano dos envolvidos. Dessa maneira, o estudo evidenciou que o estabelecimento de protocolos de diálogos, gerarão uma comunicação ativa que culminará em uma assistência de qualidade (WITTENBERG E, et al., 2019).

Ademais, foi ressaltado, que em relação às más notícias, a comunicação é um ato difícil e complexo, gerador de sentimentos de ansiedade e estresse, para a maioria dos enfermeiros pelos aspetos emocionais, relacionais e técnicos que lhe estão associados. Atualmente no serviço, os enfermeiros estão constantemente em contato com a transmissão de más notícias, ao longo da doença incurável, sendo uma das tarefas mais difíceis com que são confrontados, o que pode gerar ansiedade e estresse ao profissional (FONTES CMB, et al., 2017).

A transmissão de más notícias são situações que geram perturbação quer na pessoa que recebe a notícia, como na que a transmite. Trata-se de um assunto considerado pelos profissionais de saúde como uma tarefa difícil, que encaram "como um calvário, fazendo-os sentir-se impotentes, ineficazes e desesperados" (SISK BA, et al., 2020).

Fontes CMB, et al. (2017), evidenciaram desafios tocantes quanto a dificuldade em reações emocionais, negação ou raiva podem ser direcionados para os prestadores de cuidados ou o próprio medo de eliminar a esperança. Para esses profissionais, uma das questões complexas, é lidar com as emoções dos doentes, como a negação e a raiva, que quase sempre é direcionada para os profissionais de saúde. Outra questão se relaciona aos comportamentos de bloqueio, em que o doente não se abre à interação, nem à revelação das suas preocupações, o que dificulta uma melhor comunicação.

Relacionando o estudo proposto por Schwappach DLB e Gehring K (2014), em que os fatores que afetaram a decisão da equipe de oncologia arrolaram a expressão das preocupações de segurança, foi evidenciado uma visão aprofundada dos medos e condições que conduzem ao silêncio e a sonorização dos envolvidos (estudo 9).

Dentre os nove estudos inseridos, 7 investigações $(77,7 \%)$ enfatizaram que a falta de treinamento e capacitações transculturais, a ausência de sistematização, falta de habilidades adquiridas na graduação, ineficácia de esforções colaborativos, aproximação e reforço nos padrões éticos, humanos e assistenciais e questões culturais podem expor uma determinada dissonância entre a autonomia profissional relacionada à equipe clínica responsável pelo tratamento oncológico (WITTENBERG E, et al., 2019; ARAÚJO BN, et al., 2017; FONTES CMB, et al., 2017; ZUCA A, et al., 2017; WEBER O, et al., 2013; MOORE PM, et al., 2013)

De acordo com Wittenberg $\mathrm{E}$, et al. (2019), a necessidade de treinamento de comunicação dos enfermeiros oncológicos se apresenta como dimensão emergencial, uma vez, que desconfortos e dificuldades comunicativas acaba se transformando em prejuízos. Schimiguel J, et al. (2015), corroboraram com o exposto e evidenciaram que o acolhimento é um comprometimento de resposta às carências dos cidadãos que procuram serviços de saúde como uma proposta que visa a melhoria das relações destes serviços com o usuário, um encontro em que há uma escuta, um processamento e a procura de resolução possível. 
A conscientização da equipe de enfermagem, assim como o paciente e seus familiares (que possuem conhecimentos leigos neste contexto), não apresentou, de acordo com os autores familiaridade com o mundo hospitalar. No entanto, a atenção concedida no momento da orientação é de grande importante, as dúvidas, sejam elas, mínimas ou complexas, precisam ser tratadas para que a segurança seja conservada no ambiente, lembrando que a utilização de um vocabulário propício, levará à fácil compreensão, dentro do contexto socioeconômico e cultural do paciente e os envolvidos, pois a orientação repassada de forma complexa pode não ser compreendida pelos ouvintes (SILVA RCV e CRUZ EA, 2011).

$O$ treinamento da enfermagem oncológica, se constitui de extrema necessidade e o desenvolvimento curricular deve ser congruente com os papéis comunicativos institucionais dos enfermeiros oncológicos. A destreza na comunicação aumenta a consciência do profissional sobre o tratamento do paciente, entendimento dos procedimentos pela família e melhora a habilidade para procedimentos, concomitantemente a confiança (COYLE N, et al., 2015; MILIC MM, et al., 2015).

Gomez-Urquiza JL, et al. (2016), ressaltaram que as instituições precisam desenvolver e oferecer intervenções destinadas à redução do esgotamento emocional, a fim de apoiar na construção de habilidades de comunicação do enfermeiro. 0 estudo 8 , buscou avaliar se o treinamento de habilidades de comunicação (CST) é eficaz na mudança de comportamento dos profissionais de saúde (HCPs), assim como no tratamento do câncer e na melhoria do bem-estar do profissional de saúde, saúde do paciente, status e satisfação. $O$ mesmo, conclui que não está claro se as habilidades adquiridas pelos profissionais de saúde são mantidas a longo prazo. Além disso, não especificou se a duração e intensidade do CST é mais eficaz e se os workshops possuem a capacidade de melhorar o impacto do CST.

Na comunicação de informações ao paciente e sua família, a atuação da enfermagem ocorre de forma ativa na ação, e a habilidade em utilizá-las na comunicação de más notícias faz parte de uma importante competência a ser desenvolvida pelos profissionais (SILVA MPJ, 2012). De acordo com o estudo Fontes CMB, et al. (2017) com a classificação número 4, objetivaram descrever o estabelecimento do processo de comunicação de más notícias e identificar como os enfermeiros o praticam, evidenciou-se, que o repasse de más notícia possui forte influência cultural no trabalho da enfermagem em países desenvolvidos, uma vez que possuem maior autonomia na discussão de notícias com os pacientes; Fontes CMB, et al. (2017) ressalta também a necessidade da clareza na linguagem utilizada pelos profissionais responsáveis pela comunicação, uma vez que o modo e a habilidade do enfermeiro, influenciará a reação do paciente no que diz respeito a mensagem, e que por vezes os pacientes não possuíam real conhecimento de sua doença e seu estado.

No estudo 5, proposto por Zuca A, et al. (2017), corrobora com o fato de que os provedores de saúde podem não estar cientes das necessidades ou preferências de seus pacientes, e os mesmos relutam em expor suas preocupações. Consequentemente, o primeiro passo para garantir a qualidade do atendimento é perguntar ao paciente sobre $o$ atendimento que ele gostaria. Conclui-se que melhorias na prestação de cuidados podem ser feitas se a equipe clínica eliciar mais regularmente os sintomas emocionais do paciente e suas preferências quanto ao envolvimento de familiares/amigos e participação na tomada de decisões médicas.

Pesquisar sobre dificuldades de cuidados aos pacientes entre enfermeiros oncológicos, tanto no quesito cultural como linguístico, foi o objetivo de Weber $O$, et al. (2016), no estudo número 6 . Os enfermeiros entrevistados, relataram dificuldades de comunicação em encontros com pacientes cultural e linguisticamente diversos. Eles consideraram a ausência de materiais bibliográficos línguas estrangeiras e a ausência de uma linguagem comum compartilhada com os pacientes. Os resultados expostos no presente estudo poderão auxiliar a enfermagem, com relação às tomadas de decisão e evidenciou que o treinamento transcultural deve ser oferecido para enfermagem oncológica (WEBER O, et al., 2016).

Indivíduos com câncer, suas famílias e cuidadores constituem uma parcela da população que apresentaram alta prevalência de estresse psicológico, o que poderia ser minimizado pela comunicação e apoio efetivos dos profissionais de saúde que prestam a assistência (MOORE PM, et al., 2013). O estudo de número 8, proposto por Moore PM, et al. (2013), indicou que as habilidades de comunicação não melhoraram 
de forma confiável com a experiência, portanto, um esforço considerável deveria ser dedicado aos cursos de atualização e especializações que poderiam melhorar as habilidades de comunicação para profissionais de saúde, principalmente envolvidos no tratamento do câncer.

Todos os estudos analisados relataram a importância da equipe de enfermagem oncológica no tratamento, reabilitação ou cuidados paliativos de pacientes com câncer. A atuação da equipe foi apresentada como peça fundamental na qualidade do repasse das informações, condutas terapêuticas e assistência humanizada para o paciente oncológico e seus familiares (ARAÚJO BN, et al., 2017). As expectativas são inúmeras e possuem abrangências positivas que vão além das dificuldades apresentadas nessa investigação. $A$ abrangência dos profissionais se apresentou como promissoras, entretanto o desenvolvimento de habilidades específicas, se fazem necessárias (WITTENBERG E, et al., 2017).

As perspectivas discorreram principalmente quanto às áreas de tecnologia e inovação com a ampliação das possibilidades do desenvolvimento de um perfil para a atuação multiprofissional e a fixação da identidade necessária para a ação diante da realidade das organizações atuais (WEBER O, et al., 2016). O desenvolvimento de ações educativas e o aprimoramento da formação, consequentemente, serão refletidos na equipe de trabalho em saúde e na comunidade assistida, como podemos ver no estudo Weber $\mathrm{O}$, et al. (2016) onde os enfermeiros relatam a dificuldade de cuidar de pacientes com línguas diferentes.

\section{CONSIDERAÇÕES FINAIS}

A comunicação de notícias na enfermagem oncológica, apresentou diversos vieses e deficiências. A incipiência de treinamento e capacitação, a ausência de sistematização, a falta de habilidades adquiridas na graduação, a improficuidade de esforços colaborativos e a aproximação nos padrões éticos, humanos e assistenciais foram os principais pontos relatados. As equipes de enfermagem são essenciais na comunicação prognóstica e possuem um relevante papel na qualidade da informação e humanização do processo, entretanto capacitações frequentes se fazem necessárias. Preocupação acerca da segurança da equipe profissional e dos pacientes, evidenciaram uma visão aprofundada dos medos e condições que conduzem ao silêncio e a sonorização dos envolvidos. A equipe de enfermagem oncológica se apresenta como indispensável na comunicação de qualidade, contudo, uma maior autonomia aos profissionais, investimentos em treinamentos e sistematizações compõe um rol de desafios e ao mesmo tempo, conduzem os profissionais às inúmeras perspectivas futuras.

\section{REFERÊNCIAS}

1. ARAUJO BN, et al. Acolhimento do enfermeiro aos familiares de portadores de câncer: a percepção do familiar. Revista Saúde e Desenvolvimento, 2017; 11: 144-55.

2. BORDIGNON M, et al. Oncology nursing professional's job satisfaction and dissatisfaction in Brazil and Portugal, 2015; 24: 925-33.

3. BRIXEY L. The difficult task of delivering bad news. Dermatology nursing / Dermatology Nurses' Ass

4. COYLE N, et al. Discutindo a morte, o morrer e os objetivos do cuidado no fim da vida: Um módulo de treinamento de habilidades de comunicação para enfermeiras oncológicas. Clinical Journal of Oncology Nursing, 2015; 19:697-702.

5. FALCÃO VM, et al. Perfil da assistência de enfermagem prestada a pacientes oncológicos, na percepção dos acompanhantes, 2020; 6: 54073-84.

6. FONTES CMB, et al. Communicating bad news: an integrative review of the nursing literature. Rev Bras Enferm, 2017; 70: 1089-95.

7. GALVÃO CM. Níveis de evidências. Acta Paul Enfermagem, 2006; 19(2).

8. GOMEZ-URQUIZA JL, et al. Prevalência, fatores de risco e níveis de burnout entre enfermeiros de oncologia: uma revisão sistemática. Oncology Nursing Forum, 2016; 43: 104-120.

9. HORLAIT M, et al. How multidisciplinary are multidisciplinary team meetings in cancer care? An observational study in oncology departments in Flanders, Belgium. J Multidiscip Healthc. 2019; 12: 159-167.

10. INSTITUTO NACIONAL DO CÂNCER (INCA). Estimativas da incidência e mortalidade por câncer. 2020. Disponível em: https://www.inca.gov.br/publicacoes/livros/estimativa-2020-incidencia-de-cancer-no-brasil. Acessado em: 4 de jun. de 2021. 
11. MELNYK BM, FINEOUT-OVERHOLT E. Making the case for evidence-based practice in nursing \& amp; healthcare. A guide to best practice. Philadelphia: Lippincot Williams \&amp; Wilkins, 2005. p.3-24.

12. MENDES JLV, et al. Importância da comunicação para uma assistência de enfermagem de qualidade: Uma revisão integrativa. Brazilian Journal of Surgery and Clinical Research - BJSCR, 2020; 32: 169-174.

13. MILIC MM, et al. Comunicar-se com os familiares dos pacientes e médicos sobre o prognóstico e os objetivos do atendimento. American Journal of Critical Care, 2015; 24: e56-64.

14. MIRANDA GM et al. Sistema informatizado à decisão clínica em enfermagem: uma construção e validação na oncologia. Enfermagem em Foco, 2019; 10(7).

15. OLIVEIRA AEG, CURY VE. Cuidar em oncologia: uma experiência para além do sofrimento. Memorandum: Memória e História em Psicologia, 2016; 31: 237-258.

16. PENA MM, MELLEIRO MM. Eventos adversos decorrentes de falhas de comunicação: Reflexões sobre um modelo para transição do cuidado. Rev Enferm UFSM, 2018; 8(3): 616-625.

17. SALCI MA, MARCON SS. Enfrentamento do câncer em família. Texto contexto - enfermagem, 2011; 20(spe): 178186.

18. SCHIMIGUEL J, et al. O acolhimento de pacientes oncológicos: uma revisão bibliográfica. Saúde em Revista, 2015; 5: 47-57.

19. SCHWAPPACH DLB, GEHRING K. Trade-offs between voice and silence: A qualitative exploration of oncology staff's decisions to speak up about safety concerns. BMC Health Services Research, 2014; 14(1): 1-10.

20. SILVA MF, BEZERRA MLR. Atuação do enfermeiro no atendimento aos cuidados continuados na oncologia. Revista JRG de Estudos Acadêmicos, 2020; 3(6): 123-137.

21. SILVA MJP. Comunicação de Más Notícias. Mundo Saúde, 2012; 36(1): 49-53.

22. SILVA RCV, CRUZ EA. Planejamento da assistência de enfermagem ao paciente com câncer: Reflexão teórica sobre as dimensões sociais. Esc. Anna Nery, 2011; 15(1): 180-185.

23. SISK BA, et al. Teamwork in prognostic communication: Addressing bottlenecks and barriers. Pediatric Blood Cancer, 2020; 67(5): 1-4.

24. SOUZA FLN, et al. A desesperança após o diagnóstico oncológico e a atuação da enfermagem. Revista Multidisciplinar em Saúde, 2021; 2(3): 04-04.

25. WALDOW VR, BORGES RF. Cuidar e humanizar: relações e significados. Acta paul. enferm., 2011; 24(3): 414-418. 